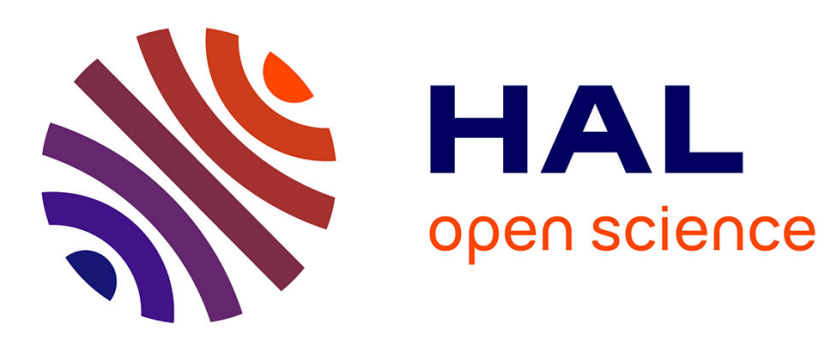

\title{
Négociation de la relation et processus d'appropriation en classe de langue
}

\author{
Violaine Bigot
}

\section{To cite this version:}

Violaine Bigot. Négociation de la relation et processus d'appropriation en classe de langue. Acquisition et Interaction en Langue Etrangère, 2005, Interactions et diversité des conduites d'apprentissage, 22, pp.17-45. hal-01319720

\section{HAL Id: hal-01319720 \\ https://hal.science/hal-01319720}

Submitted on 25 May 2019

HAL is a multi-disciplinary open access archive for the deposit and dissemination of scientific research documents, whether they are published or not. The documents may come from teaching and research institutions in France or abroad, or from public or private research centers.
L'archive ouverte pluridisciplinaire HAL, est destinée au dépôt et à la diffusion de documents scientifiques de niveau recherche, publiés ou non, émanant des établissements d'enseignement et de recherche français ou étrangers, des laboratoires publics ou privés. 


\section{NEGOCIATION DE LA RELATION ET PROCESSUS D'APPROPRIATION EN CLASSE DE LANGUE. \\ Violaine BIGOT \\ (Université d'Angers, Diltec, Kachina) ${ }^{1}$}

Résumé :

Nous étudions ici la manière dont un étudiant adulte, filmé dans plusieurs cours de langue, prend des initiatives interactionnelles qui détournent le cours des échanges et du scénario didactique prévu par l'enseignante. Les extraits analysés montrent comment, à travers ces offensives discursives, l'étudiant renégocie la relation professeur-étudiant initiée par l'enseignant et redéfinit son territoire d'apprenant de manière à développer des séquences où ses productions langagières et l'étayage qu'il sollicite sont susceptibles de favoriser le développement de ses compétences langagières. (mots clefs : interaction didactique, relation interpersonnelle, acquisition de L2, focalisation métalinguistique.)

\section{Introduction}

L'étude présentée ici s'appuie sur un corpus vaste déjà largement exploité dans le cadre d'une recherche orientée sur les modes de construction de la relation interpersonnelle en classe de langue. Nous nous concentrons ici sur un des étudiants ${ }^{2}$ de ce corpus En introduction sont présentés : le corpus dont nos données sont issues, un bref cadrage méthodologique sur le traitement de ces données et les questions auxquelles cette étude tente de répondre ${ }^{3}$.

\subsection{Historique du corpus}

La construction de la relation interpersonnelle en classe de langue a fait l'objet de ma recherche doctorale. Le projet était d'étudier les comportements de cinq enseignants placés dans des contextes tout à fait similaires : même institution, même type d'étudiants voire mêmes étudiants, mêmes types d'activités didactiques. Je voulais étudier la diversité des 'lieux' de construction de cette relation et évaluer la marge de manœuvre dont disposent les enseignants. Les analyses étaient focalisées sur les comportements langagiers des enseignants et tentaient de montrer dans quelle mesure les positionnements énonciatifs de leurs productions discursives, la manière dont ils catégorisent verbalement les différents partenaires du cours, la manière dont ils structurent, clôturent et ouvrent les séquences, la manière dont ils font circuler la parole et dont ils se posent en garant et gérant de la norme contribuent à définir les rapports de place entre participants et plus généralement à construire une relation interpersonnelle professeur-élève spécifique. Cette étude était donc volontairement «partiale » puisque le projet était d'étudier le rôle spécifique de l'enseignant dans la construction de cette relation.

\subsection{Présentation des données et du cadre méthodologique d'analyse}

On peut considérer, à la suite de Arditty et Vasseur, que le linguiste interactionniste doit non seulement, en tant que sujet parlant, «mobiliser une connaissance intuitive des phénomènes

\footnotetext{
${ }^{1}$ violaine.bigot@univ-angers.fr, Université d'Angers, 11 bvd Lavoisier, F 49045 Angers Cedex 01.

${ }^{2}$ J'utilise le terme «étudiant » pour désigner le locuteur par la principale caractéristique du statut activée dans l'échange didactiques, sans a priori sur son engagement dans une activité d'apprentissage. Le terme « apprenant » est réservé aux citations et aux analyses qui portent sur des passages où l'attention est focalisée sur le travail d'appropriation.

${ }^{3}$ Je remercie Jo Arditty pour toutes les discussions que nous avons eues autour de ces données et pour ses relectures et commentaires de cet article.
} 
socio-langagiers qui lui permette de se mettre à la place des participants » mais qu'il doit aussi appuyer ses décisions d'interprétation sur «d'autres critères: l'examen précis des formes utilisées (linguistiques mais aussi paralinguistiques, gestuelles, kinésiques...) de la manière dont elles sont reprises ou non par leur auteur ou ses partenaires, plus globalement des réactions qu'elles provoquent chez ces derniers, de l'aboutissement ou non des actions entreprises, de leur scansion, de la façon dont l'interaction est structurée et, éventuellement menée à son terme etc. » (Arditty et Vasseur, 1999 : 8-9).

Le corpus présente dès lors un double avantage. D'une part sa longueur (une quinzaine $\mathrm{d}^{\prime}$ 'heures ${ }^{4}$ ) facilite la mobilisation par le chercheur, de son intuition de locuteur et l'adoption du point de vue des participants. D'autre part, le fait que trois de ces quinze heures ont fait l'objet d'une transcription fine permet de procéder à «l'examen précis » évoqué ci-dessus et de porter un regard distancié sur les données. On peut ainsi «faire le tour » des données en s'appuyant éventuellement sur un traitement quantitatif de certains phénomènes. Enfin, des passages isolés peuvent être étudiés en détail sans pour autant perdre de vue le contexte et le co-texte dont ils sont extraits.

Le corpus sur lequel porte la présente analyse est donc constitué d'une quinzaine d'heures de cours vidéoscopés, dans lesquels un même groupe d'étudiants, composé de 12 stagiaires originaires de 5 pays différents, travaillent avec deux enseignantes différentes : Viviane et Dorie. Les activités sur lesquelles portent les analyses sont d'une part des activités de présentation (premiers moments du premier cours entre le groupe et chacune des deux enseignantes, que nous appelons « séquences d'ouverture ») et des activités de compréhension de textes (articles de presse avec Viviane et documentaire télévisé avec Dorie).

\subsection{Présentation de l'étude}

Ce sont donc les liens entre les procédés de négociation de la relation et les activités d'appropriation et de développement de la compétence langagière dans lesquels s'engage un des étudiants du corpus (Hamed) qui seront explorés dans cet article. La présente étude s'inscrit pleinement dans la reconnaissance du caractère à la fois négocié et co-construit de «l'ordre des relations sociales et des contenus scolaires »dont Mondada et Pekarek nous rappellent qu'elle est importante dans la mesure où elle "montre qu'une tâche n'est pas déterminée par un curriculum ou un programme, ni par l'ingéniosité pédagogique du seul responsable de la classe mais qu'elle est accomplie de façon située et distribuée par tous les participants concernés » (Mondada et Pekarek, 2001 : 120). C'est le point d'articulation entre construction de la relation entre participants et co-définition de la tâche que nous allons explorer dans cette étude.

Dans un premier temps, pour faciliter la contextualisation des données qui seront ensuite analysées, les modes de construction de la relation interpersonnelle privilégiés par les deux enseignantes des cours auxquels participe Hamed, seront brièvement présentés à partir de quelques résultat de ma recherche doctorale.(2.1.). Puis ce sont les interventions de Hamed que nous caractériserons globalement pour expliquer pourquoi la recherche s'est centrée sur lui (2.2).

Dans la section 3, nous étudierons quelques extraits où les interventions d'Hamed peuvent être interprétées comme des formes de renégociation de la relation plus ou moins imposée par

\footnotetext{
${ }^{4}$ Rappelons que cette quinzaine d'heures fait partie d'un corpus plus large de 33 heures d'enregistrements faisant intervenir d'autres enseignants et d'autres groupes d'apprenants. L'ensemble de ce corpus a été découpé en séquences, une bonne partie a fait l'objet de transcriptions rapides. Un certain nombre d'activités didactiques ont donné lieu à une transcription fine. Le choix de ces activités a été déterminé de manière à assurer une certaine «comparabilité » des données (activités de classe de même nature : présentation des étudiants lors d'un premier cours et activités de compréhension de textes). Les trois heures de transcriptions sur lesquelles porte les analyses de cet article font partie de ce corpus clos.
} 
l'enseignant. Nous verrons ensuite (section 4) que ses prises de parole lui permettent aussi de contribuer à la négociation des tâches en cours, et d'orienter le travail métalinguistique collaboratif. Enfin nous nous intéresserons aux interventions de Hamed dans leur dimension figurative et notamment, nous verrons comment il cherche non sans difficultés, à se construire une 'image d'étudiant modèle' (section 5).

\section{Présentation des informateurs}

\subsection{Les partenaires enseignantes}

Les deux enseignantes avec lesquelles Hamed interagit dans ce corpus ont donc fait l'objet d'une étude approfondie sur les modes de construction de la relation. Nous en donnons ici un bref compte-rendu pour situer le cadre dans lequel se situent les échanges que nous étudierons.

Viviane, jeune enseignante au statut précaire, est apparue, dans les cours enregistrés et selon les divers critères d'analyse que je m'étais donnés, comme une enseignante qui privilégie un mode de construction de la relation inégalitaire et fortement hiérarchisé. D'un point de vue énonciatif, elle articule tous les échanges de présentation du cours autour d'une opposition Moi-l'enseignante ${ }^{5}$ (mes attentes, mes besoins, mes exigences) / Vous-l'entité groupe (vos droits et vos devoirs, vos mauvaises habitudes). Alors que son groupe d'étudiants comprend essentiellement des adultes déjà engagés dans la vie active, et notamment deux enseignants d'université, elle ne les catégorise que comme des étudiants ( je ne veux pas voir les étudiants...) et ne leur donne quasiment jamais l'occasion 'd'activer' d'autres caractéristiques de leur statut. Elle-même ne se catégorise que comme enseignante. Par ailleurs, l'occupation du temps de parole, le recours aux questions didactiques ainsi que les modes de correction privilégiés ont été comparés, grâce à un traitement quantitatif des interactions des séquences d'ouverture. Il apparaît que Viviane a le taux d'occupation du temps de parole ${ }^{6}$ le plus élevé ( $94,8 \%$ contre $85,8 \%$ chez Dorie et $74,1 \%$ chez une troisième enseignante), que son recours aux questions didactiques ${ }^{7}$ est très fréquent (22 questions didactiques contre 1 et 0 chez ses deux collègues pour des activités comparables) et qu'elle procède avant tout à des corrections non intégrées communicativement ${ }^{8}$ et fortement marquées didactiquement.

Du point de vue des catégorisations ${ }^{9}$, Dorie ne se catégorise que comme enseignante mais elle invite les étudiants à se catégoriser de diverses manières en leur donnant à chacun un temps de présentation (traditionnel 'tour de table'). Elle catégorise d'ailleurs elle même les étudiants lorsqu'elle dispose d'informations valorisantes que les étudiants n'ont pas données spontanément. Ainsi, après que Hamed a expliqué qu'il vient poursuivre sa formation en France, c'est elle qui informe le groupe qu'il est professeur de karaté.

Pour résumer les analyses détaillées que nous avons conduites sur les modes de construction de la relation privilégiés par ces enseignantes, nous pouvons reprendre la distinction entre

\footnotetext{
${ }^{5}$ Un moi qu'elle associe également par un jeu lexical et syntaxique au reste de l'équipe enseignante.

${ }^{6}$ La comparaison porte sur Viviane-séquence d'ouverture, Dorie-séquence d'ouverture et une troisième séquence d'ouverture entre une autre enseignante et un autre groupe. Le calcul est opéré en nombre de mots sur les trente premières interventions de ces trois séquences d'ouverture

${ }^{7}$ Egalement appelées display question dans la terminologie anglo-saxonne, les questions pédagogiques - ou questions didactiques - se caractérisent par le fait qu' «elles sont posées à des fins de contrôle, et leur fonction essentielle semble être de vérifier si les élèves fonctionnent ou non dans le cadre de référence de l'enseignant » (Grandcolas $1980: 54)$.

${ }^{8}$ Rappelons que de Pietro, Matthey et Py $(1989$ : 114) parlent de correction « communicativement intégrée » lorsque la focalisation métalinguistique n'est pas clairement marquée (pas d'accent d'intensité ou de décontextualisation du syntagme corrigé par exemple). On peut dire que dans ce cas la fonction de tutelle pour communiquer assumée par le locuteur natif-enseignant prime la fonction de tutelle pour apprendre (Dausendschön-Gay et Krafft 1994, Bigot 2002a).

${ }^{9}$ Sur l'analyse des procédés de catégorisation en classe de langue voir notamment le chapitre 4 de Mondada et Gajo (2000).
} 
l'axe vertical qui est celui des relations hiérarchiques et des rapports de pouvoir et l'axe horizontal qui est celui de la distance-intimité, de la familiarité. Cette opposition théorique proposée par Kerbrat-Orecchioni (1992), pour l'étude des relations interpersonnelles ne doit pas faire perdre de vue que l'analyse des rapports de place entre les participants d'une interaction-occurrence ne peut pas être dissociée de l'analyse des rapports de distance/familiarité et notamment des procédés de politesse mis en œuvre. Les comportements langagiers de Viviane évoqués ci-dessus, contribuent donc à la construction d'une relation verticale marquée par un rapport de place assez fortement inégalitaire. Elle produit en effet de nombreux marqueurs de places ou taxèmes (Kerbrat-Orecchioni,1992: 71 et suiv.) qui rappellent et renforcent la relation hiérarchisée enseignant-étudiant. Le mode de développement de la relation horizontale (maintien à distance des participants) contribue à la construction de ce rapport de place hiérarchisé.

Dorie produit très peu de marqueurs de place renforçant la position haute que lui confère son statut d'enseignant et ses modes de sollicitation ou de corrections, tendent plutôt à rééquilibrer la relation. Du point de vue de la relation horizontale qu'elle développe, il est frappant de constater que si elle donne aux étudiants des occasions de se catégoriser de multiples manières, favorisant ainsi la création de liens, notamment entre eux, elle se tient elle-même en retrait de ce tissage de liens. ${ }^{10}$

\subsection{Le choix d'Hamed comme sujet d'étude}

Dans notre corpus de thèse interviennent quatre groupes d'étudiants différents qui représentent un total de 42 étudiants de 14 nationalités différentes. Ceux qui ont lu ce corpus ont tous été frappés cependant par les interventions d'un de ces étudiants, qui se définit luimême, du point de vue de son appartenance nationale, comme un israëlien-arabe : Hamed.

Il est difficile, sans anticiper sur l'analyse des données, de cerner ce qui rend ces interventions remarquables. On peut noter cependant qu'elles sont fréquentes. A titre d'exemple, on peut ainsi indiquer que sur cent interventions prélevées ${ }^{11}$ dans deux cours donnés par deux enseignantes différentes, il intervient 18 fois dans le premier et 14 dans le second. Or le groupe comprend 12 étudiants qui ensemble n'interviennent que 28 et 30 fois $^{12}$. Hamed intervient souvent lorsque l'enseignant pose des questions à la cantonade et, phénomène plus rare, il intervient spontanément, sans qu'aucune intervention étudiante n'ait été sollicitée ni même que l'enseignant ait donné de signe clair de fin de tour de parole. C'est ce qu'on peut appeler des 'interventions spontanées'. On compte ainsi 4 interventions spontanées sur les 18 de la première séquence étudiée, et 2 sur ses 14 interventions dans la seconde séquence. Enfin, ses interventions rompent souvent avec le caractère consensuel des interactions qui prévaut le plus souvent en classe de langue. Nous reviendrons plus loin sur les différents registres d'ironie ou parfois de claire contradiction auxquels Hamed fait appel et qui donnent

\footnotetext{
${ }^{10}$ Il est intéressant de noter que la troisième enseignante sur laquelle porte notre étude présente un troisième profil. Comme Dorie, elle produit peu de taxèmes rappelant sa position haute d'enseignante. Elle produit même des taxèmes de position basse favorisant le rééquilibrage de la relation. Mais elle crée parallèlement les conditions favorables à un rapprochement sur l'axe horizontal. Elle favorise les catégorisations multiples des apprenants et elle se catégorise elle-même de multiples manières, favorisant les rapprochements par l'appartenance à des catégories communes (par exemple, à deux étudiantes qui disent qu'elles sont allemandes, elle explique que sa fille vient justement de partir pour un an en Allemage, produisant ainsi une catégorie commune des gens qui ont des liens privilégiés avec l'Allemagne.).

${ }^{11}$ Il s'agit d'une activité de 'remue-méninge' sur l'emploi du temps de la classe et d'une activité de compréhension globale d'un document vidéo. Ont été retenus, dans les deux premiers cours des deux enseignantes, les premières séquences d'au moins cent interventions où la circulation de la parole n'est pas de type 'tour de table' (ce qui n'aurait pas vraiment permis d'observer le taux de participation d'Hamed). Dans ces séquences les enseignantes posent presque exclusivement des 'questions à la cantonade'.

${ }^{12}$ Les réponses collectives où les apprenants ne sont pas identifiés n'ont pas été comptabilisées.
} 
à ses interventions un caractère non-préférentiel, non-attendus ${ }^{13}$. On note cependant que le mode plus ou moins agonal sur lequel se développent les échanges entre Hamed et les enseignantes n'empêche pas le maintien d'activités langagières orientées vers l'appropriation de la langue.

Nous essaierons donc, non seulement de comprendre ce qui rend les comportements de Hamed 'remarquables' mais aussi et surtout ce qui se joue en termes d'appropriation langagière, dans ces échanges particuliers entre locuteur natif et locuteur non-natif en contexte didactique.

\section{Négociation et renégociation de la relation :}

\subsection{Tentative de putsch relationnel :}

Pour entrer dans les données nous étudierons une séquence située tout au début du premier cours de Viviane. Hamed y produit plusieurs «interventions spontanées » (22, 24, 26 28) qui bousculent momentanément le rythme des échanges. Le groupe d'étudiants qui participe à ce cours a été partiellement refondu avec d'autres étudiants trois jours avant ce cours. A cette occasion, l'emploi du temps a été légèrement modifié. Viviane, n'intervient que de manière sporadique dans l'établissement et, pendant la période où a été recueilli le corpus, elle ne donne qu'un seul cours, le mercredi, cours pendant lequel elle a été filmée. Pendant tout le début de la séquence d'ouverture de ce premier cours, elle reprend l'emploi du temps du groupe en posant aux étudiants des 'questions didactiques' sur les horaires, les enseignants et les activités qu'ils auront chaque jour de la semaine. Elle note leurs réponses au tableau.

\footnotetext{
${ }^{13}$ Ce que nous qualifions ici de «non-attendu», pourrait aussi être qualifié à la suite de Levinson de «dispreferred». Les réponses «non-préférées» sont produites avec un certain délai et leur production s'accompagne de précautions rituelles. (Kerbrat-Orecchioni, $1990: 272$ ). Il faut noter cependant que l'étude de levinson porte sur les deuxièmes éléments d'une paire adjacente (réponse au sens large). Or, dans la section 3.1, l'intervention 'non-attendu' étudiée est une intervention initiative. C'est d'ailleurs plus la réaction qu'elle suscite chez l'enseignante que ses caractéristiques propres qui rendent le caractère 'non-attendu' de cette intervention tangible.
} 
extrait 1 (Viviane cours 1$)^{14}$.

19 P. ouais de la quoi orale de la hm hm orale / qu'est-ce que vous essayez de faire

$20 \mathrm{Na}$ compréhension

21. P la compréhension orale ok <elle écrit au tableau> donc avec Dorie Graf le lundi matin / vous parlez des actualités / vous faites de la compréhension orale / ça ça ne CHANge pas $\downarrow$ d'accord $\uparrow$ ça va rester comme ça / le mardi matin ++ une bonne nouvelle / le mardi matin vous allez pouvoir DORmir $\downarrow$ d'accord $\uparrow$ parce que / il n'y aura pas cours le mardi matin ok $\uparrow \underline{\text { donc vous allez }}$

22 Hamed chaque semaine

23.P pardon $\uparrow$

24. Hamed chaque semaine comme ça maintenant

25. P d'accord donc vous allez pouvoir dormir le mardi matin $\downarrow$ c'est comme ça

26. Hamed c'est obligé non $\uparrow$

27. P c'est obligé $\uparrow$ non / vous faites c'que vous voulez hein <rire> le mercredi matin / c'est aujourd'hui / on va travailler avec moi

28. Hamed XXX (Viviane)

29. P ouais ben oui j'm'appelle Viviane pardon excusez-moi j'ai oublié <écrit au tableau $>$ mon nom c'est Pommier + mais attention $\uparrow(.$.

Précisons que les étudiants connaissent cet emploi du temps puisque, d'une part, il est très proche de celui qu'une partie du groupe a suivi depuis plusieurs semaines, d'autre part, il a été

\footnotetext{
${ }^{14}$ CONVENTIONS DE TRANSCRIPTION

<rires> <à voix basse>

bonjour <moins fort $>$ la surprise j'aime la surprise < $>$ installez-vous des JEUX

/

$+$

$\downarrow$

$\uparrow$

euh :

XXX

XX (tu viens ?)

A : comme ça je

A :C'EST DIFFICILE

$\mathrm{P}$ depuis toujours $=$

A =OUI OUI

[gest]

Af ; Am

entre $\langle>$ : description d'éléments para-verbaux ou non-verbaux.

le signe $<>$ indique la fin de portée du commentaire méta lorsque celui-ci porte sur plusieurs mots. Ici, c'est "la surprise j'aime la surprise" qui a été prononcé avec une faible intensité.

les majuscules indiquent la mise en valeur d'une ou plusieurs syllabes par un accent d'intensité et/ou par une montée brusque de la hauteur éventuellement accompagnée(s) d'un allongement.

(limites d'unités intonatives que l'on a jugé utles de noter pour faciliter la lecture

pause (++ / +++ pauses plus longues). Pour les pauses longues ( $\geq 5$ ") la durée est indiquée entre parenthèses.

la courbe intonative du groupe rythmique est globalement descendante.

la courbe intonative du groupe rythmique est globalement montante.

la syllabe est allongée.

séquence inaudible, le nombre de X correspondant à peu près au nombre de syllabes.

séquence reconstituée dont le transcripteur n'est pas certain. les mots soulignés indiquent la partie des énoncés qui se chevauchent.

A enchaîne immédiatement, sans la moindre pause sur les paroles de P.

transcription phonétique.

apprenant féminin ou apprenant masculin non identifié
} 
mis en route deux jours plus tôt. Les étudiants notamment n'ont pas eu cours la veille. Le changement qu'elle leur indique concernant le mardi n'est donc pas vraiment une 'information nouvelle'. Le caractère didactique des échanges est assez contraignant puisque l'extrait ci-dessus est précédé d'un enchaînement de cinq échanges ternaires où les brèves réponses des étudiants aux questions didactiques de l'enseignante (Madame X, parler des journaux, les actualités, on écoutait les cassettes) sont toutes suivies d'évaluation. On a un exemple de ces échanges ternaires dans les trois premières interventions de l'extrait 1.

C'est dans ce contexte qu'intervient la première 'montée au créneau' de Hamed. Dans un premier temps (24) il demande une précision qui prend la forme d'une vraie demande d'information. Il brise ainsi l'enchaînement des échanges ternaires et redonne à l'échange un caractère informatif. Cette intervention surprend aussi parce qu'elle est la première intervention initiative d'un étudiant et que Hamed coupe la parole à l'enseignante pour poser sa question (chevauchement). Dans un deuxième temps (26), il répond à la pointe d'humour de l'enseignante par une question ironique qui semble déjà stigmatiser 'l'autoritarisme' de l'enseignante dans cette séquence d'ouverture. Enfin, dans un troisième temps, il pose une question dont le caractère 'non-attendu' est révélé par les hésitations de l'enseignante pour formuler sa réponse, réponse qui, dans un autre contexte, pourrait fuser.

Le contexte dans lequel intervient cette question de Hamed mérite d'être examiné de près. En effet, dans l'énoncé qui précède (27), l'enseignante s'inscrit énonciativement dans son discours de manière ambivalente. le mercredi matin / c'est aujourd'hui / on va travailler avec moi. Le on pourrait ici s'analyser comme un 'nous inclusif' recouvrant 'moi+vous'. Le choix de ce on devrait alors dans ce cas plutôt appeler un complément du type ensemble (on va travailler ensemble) qui reprendrait l'ensemble des personnes désignées dans le sujet ou sinon, un complément désignant une 'troisième personne', hors du couple Je-Vous (on va travailler avec X). La formule on va travailler avec moi constitue ce que j'ai proposé d'appeler un 'hiatus énonciatif' ${ }^{15}$ (Bigot, 2002b). Et d'une certaine manière, par sa question, Hamed s'engouffre dans la brèche ouverte par ce hiatus pour suspendre momentanément la reprise détaillée de l'emploi du temps et solliciter de l'enseignante que l'on s'arrête un peu sur ce moi qui constitue la véritable nouveauté pour la plupart des étudiants.

Ce passage est également l'occasion d'un glissement dans la manière de désigner les enseignants. En effet, jusque là, Viviane a désigné sa collègue par prénom+nom (Dorie Graf : deux fois) et par Madame + nom de famille (Madame Graf : deux fois). Lorsqu'il s'agit de décliner son propre patronyme à l'instigation de Hamed, elle semble hésiter, donne d'abord son prénom (je m'appelle Viviane), s'excuse - alors que sa face est potentiellement plus mise à mal par la question d'Hamed que celle des étudiants par son oubli, puis elle indique son nom de famille (Mon nom c'est Pommier). Après ce coup de force de Hamed, la reprise de l'emploi du temps se poursuit et pour le cours du jeudi, on parle de Madame Graf, puis de Dorie Graf. L'enseignant du vendredi quant à lui est désigné par son prénom simple (Charles). ${ }^{16}$ Enfin, à la fin de cette séquence, l'enseignante reprend tout l'emploi du temps, et les enseignants y sont cette fois uniquement désignés par leur prénom.

\footnotetext{
${ }^{15}$ On peut exclure d'interpréter le « on » comme un « il » semblable à l'emploi spécifique des commerçants du type « et le monsieur, il prendra quoi ? ». D'une part parce que ce «on» que l'on retrouve à de nombreuses reprises pour parler des cours du mercredi devrait, s'il désignait exclusivement les étudiants, se retrouver également dans l'évocation des cours dispensés par les autres enseignants, ce qui n'est pas le cas. D'autre part, on retrouve ce «on» associé à d'autres termes qui en lèvent l'ambiguïté comme en 39: «ensemble on va travailler tous les mercredi matin donc ... »

${ }^{16} \mathrm{Il}$ est intéressant de remarquer que Charles sera désigné tout au long du cours exclusivement par son prénom (6 fois). Celui-ci, comme Viviane, est un enseignant fraîchement sorti de la formation de maîtrise de didactique des langues (moins de trois années d'expérience professionnelle) tandis que Dorie est une enseignante expérimentée qui a même été formatrice de Viviane et de Charles pendant leur année de maitrise.
} 
Les trois interventions successives de Hamed peuvent donc être interprétées comme des tentatives de briser le dialogue canoniquement didactique que l'enseignante a mis en place, dès les premières minutes de cours, par son mode de questionnement et d'évaluation. La première intervention brise ce jeu des fausses questions. La troisième semble réclamer à l'enseignante une implication, minimale certes puisqu'il s'agit seulement de décliner une identité, mais qui 'dépasse' le simple rôle statutaire qu'elle occupe depuis le début du cours. La deuxième s'appuie sur l'humour pour créer du 'jeu' dans le rouage des échanges et créer un (furtif) espace relationnel différent.

\subsection{Le recours à l'ironie}

De nombreuses interventions spontanées de Hamed ont une valeur ironique et semblent justifiées, si l'on admet qu'on ne parle pas pour ne rien dire, par la volonté de faire rire. Ainsi, comme Hamed a proposé le mot chemin alors qu'elle attend celui d'impasse, Dorie demande c'est un petit chemin oui : (...) comment on appelle ça un chemin qui est fermé. Hamed répond en riant: un chemin fermé. Deux jours plus tard, suite à un malentendu dû à la mauvaise prononciation par un étudiant vietnamien du mot 'chômage', Viviane explique la différence entre chemin et chômage : ça c'est un chemin c'est ça $\uparrow$ ça c'est une petite route $\uparrow$ d'accord $\uparrow$. Elle accompagne son explication d'un dessin. Hamed fait rire toute la classe en commentant laconiquement le dessin du zigzag par c'est une rivière Hamed fait souvent des réponses ou des commentaires ironiques de ce type. Le recours à l'humour a toujours cette 'vertu relationnelle' analysée dans l'extrait 1, dans la mesure où il permet, par l'effet de décalage qu'il génère, de suspendre momentanément la relation déterminée par les statuts des participants. Les interventions ironiques de Hamed, concourent à ce que Moore et Simon appellent la «déritualisation des scénarios d'apprentissage bâtis par les enseignants » (2002 : 133). Les phénomènes de déritualisation étudiés par ces auteures ne s'appuient pas comme ici sur un recours à l'humour mais ils prennent aussi leur origine dans des interventions apprenantes qui font sortir le cours de l'interaction didactique de son lit habituel.

Comme le souligne les auteures, ce qui est en jeu dans ce type de séquences c'est «l'expression par l'apprenant de sa double identité, celle du 'je' sujet personne et celle du 'je' apprenant pour une prise en compte de l'apprenant dans toute la complexité de ses dimensions sociale, socioculturelle et cognitive » (p.139). C'est non seulement vrai pour le recours à l'ironie mais sans doute aussi pour l'extrait 1 où, en faisant dévier l'enseignante du jeu de rôle " enseignant-élève » qu'elle avait instauré, Hamed cherche à faire entrer en scène d'autres facettes des identités de l'enseignante et des étudiants.

Nous nous sommes jusque là intéressés à la manière dont l'apprenant peut prendre des initiatives discursives et intervenir sur le développement thématique des échanges, pour créer un autre espace relationnel que celui 'autorisé' par le scénario didactique développé par l'enseignante. Nous allons maintenant nous interroger sur les liens entre ces procédés de déritualisation et les processus d'appropriation.

\section{Déritualisation et travail d'appropriation}

Les phénomènes de déritualisation étudiés par Moore et Simon sont étroitement liés à la redéfinition du territoire d'apprenant tel que Py l'a défini en 1993 autour des trois pôles : le système (qui «assure la cohésion des connaissances linguistiques »), la tâche («l'activité en langue dans laquelle l'apprenant est engagé ») et la norme (qui exerce sur le système des pressions explicites - rappel de la norme par l'enseignant par exemple - et implicite perception par l'apprenant d'un écart entre son système et celui des locuteurs natifs.) (Py, 1993 : 10). Moore et Simon expliquent notamment que les séquences où l'on observe une déritualisation du scénario didactique constituent des «moments clefs dont les orientations 
discursives, soudain prises en charge par les apprenants, leur permettent de se re-situer en tant qu'apprenant-actif, et de redessiner les contours de leur territoire tels qu'ils choisissent euxmême de le définir dans l'interaction. » (Moore et Simon, 2002 : 138).

Nous étudierons ci-dessous deux aspects du travail collaboratif d'appropriation que peuvent générer les séquences de déritualisation initiées par Hamed.

\subsection{Déritualisation du scénario, accommodation et recours à des schémas partagés}

Le projet de l'enseignante dans l'extrait 3, se trouve momentanément suspendu, car Hamed sollicite de la part de l'enseignante un étayage spécifique pour résoudre un problème apparu bien plus tôt dans le cours (extrait 2). S'accommodant à la situation, les partenaires de l'échange développent un travail collaboratif qui s'appuie sur un schéma partagé : l'exercice structural de répétition.

extrait 2 (Viviane cours 1$)$

147 Hamed Xj'ai un problème écritXXX

$148 \mathrm{P} \quad$ vous avez un problème à l'écrit je sais \ avec Charles vous allez beaucoup travailler l'écrit / aussi

149 Hamed c'est expression c'est pas

$150 \mathrm{P} \quad$ qu'est-ce que ça veut dire l'expression écrite

151 Hamed moi je connais expression ça veut dire les: un petite phrase veut dire quelque chose / par exemple / avoir [Kor] sur main

$152 \mathrm{P} \quad$ avoir quoi $\nearrow$

153 Hamed avoir [Kor] sur sur sur la main

$154 \mathrm{P} \quad$ avoir $\nearrow++$ avoir $\nearrow$ <elle dessine un cour avec ses doigts>

155 As cœur

$156 \mathrm{P} \quad$ LE coeur sur la main / si vous dites l'expression mais que vous ne la dites pas correctement / chpouf <en souriant elle fait le signe de couler avec ses deux pouces $>$ c'est raté

157 Hamed désolé

extrait 3 (Viviane cours 1)

$\begin{array}{lll}835 & \text { P } & \text { ok } \downarrow \text { le mot que vous trouvez difficile à prononcer } \\ 836 & \text { Hamed } & \text { le }[\text { kor] le cœur le cœur ou XXX la lettre o e } \\ 837 & \text { P } & \text { e e } \\ 838 & \text { Hamed } & \text { e ouais par exemple le cœur } \\ 839 & \text { P } & \text { le cœur } \\ 840 & \text { Hamed } & \text { cœur } \\ 841 & \text { P } & \text { peur } \\ 842 & \text { Hamed } & \text { peur oui } \\ 843 & \text { P } & \text { beurre } \\ 844 & \text { Hamed } & \text { beurre } \\ 845 & \text { Af } & \text { sœur } \\ 846 & \text { Hamed } & \text { sœur ça va ça va }\end{array}$




\section{$847 \mathrm{P} \quad$ oui donc c'est le son [œ] et avec le son o le corps l'école \\ 848 Hamed oui l'école ça va cœur et beurre [ker] ou [ber]}

Le premier extrait intervient dans une séquence où l'enseignante demande aux étudiants quelles sont leurs attentes pour le cours. Hamed explique qu'il souhaite travailler l'écrit. La mauvaise prononciation du mot cœur déclenche une séquence de focalisation sur la forme. L'enseignante opère une sollicitation d'auto-correction dont le but semble plus d'inciter Hamed à rapprocher son interlangue du système que de lever un éventuel malentendu puisque la symbolisation par l'enseignante du cœur avec les doigts montre qu'elle a compris. On peut donc considérer que l'enseignante impose sa définition du territoire d'apprenant en opérant une focalisation étroite sur le pôle norme et en suspendant temporairement la tâche en cours qui consistait à recenser les besoins des étudiants.

Dans l'extrait suivant, qui intervient plus tard dans le même cours, les rôles sont renversés. Alors qu'il est en train de répondre à une sorte de «questionnaire de Proust » où il doit se définir à travers des mots de la langue française qu'il aime, qui lui font peur, qui le rendent heureux, qu'il trouve difficile à prononcer etc., Hamed en profite pour se donner l'occasion de repratiquer le mot qui a déclenché la séquence de focalisation métalinguistique au début du cours (extrait 2). Il ne se contente pas d'indiquer le mot mais isole dans ce mot ce qu'il pense être à l'origine de la difficulté (rapport graphie-phonie œ/[œ]). Il détourne donc sensiblement l'activité de son but initial: se présenter au groupe à travers un lexique personnel. L'enseignante accepte ce glissement de scénario et collabore dans une longue séquence essentiellement dialogale. En 839 elle répète le cœur initiant ainsi une séquence qui a des allures d'exercice de répétition phonétique. On peut même penser que la forme 'exercice structural' offre ici une ressource commune aux participants qui facilite leur travail de collaboration. Hamed en 840 , non seulement répète le terme mais le décontextualise complètement, renforçant ainsi la centration sur le niveau phonétique. L'enseignante en 841 et 843 reprend l'initiative en proposant d'autres mots à répéter mais elle conserve le 'format' proposé par Hamed en 840 puisqu'elle donne les mots à répéter sans déterminant. L'intervention d'une apprenante, qui en 845 , propose un autre mot à répéter (sœur) montre que la collaboration dans cette exercice ad hoc de répétition phonétique s'étend à une partie au moins du groupe d'apprenants. On peut se demander si c'est cette intervention apprenante qui motive Hamed à tenter de mettre un terme à la séquence, prenant ainsi en charge une des fonction spécifiques de l'enseignant à savoir la structuration des échanges et le bornage des séquences (Bange 1992). Quoi qu'il en soit, la séquence ne se termine pas là et se poursuit pendant 11 interventions au delà de l'extrait reproduit. En effet, suite au refus de Hamed de travailler sur les mots contenant un o ouvert, l'enseignante recentre le travail sur les mots 'peur', 'cœur' et 'sœur', autant de mots que Hamed répète en écho à un rythme qui s'accélère. On peut noter au passage que le mot sœur, lorsqu'il est présenté par l'enseignante, est répété sans commentaire. Grâce à une collaboration étroite et un travail d'ajustement réciproque serré, Hamed a eu l'occasion de pratiquer un des points de son système phonologique qu'il considère comme hypothétique.

\subsection{La déritualisation et ses limites :}

Nous voulons revenir ici sur quelques interventions ironiques de Hamed et sur les conditions nécessaires pour qu'une initiative discursive d'apprenant puisse donner lieu à un travail d'appropriation. Nous avons donc fait l'hypothèse que par ses remarques Hamed cherche non seulement à faire rire le groupe mais qu'il poursuit également son travail d'appropriation langagière. 
Dans l'extrait 4, qui intervient dans la séquence où l'enseignante demande aux étudiants leurs attentes sur le cours, on assiste à un retour de Hamed sur le devant des échanges. Après avoir demandé à faire de l'expression écrite (cf. extrait 2), il demande à faire de la grammaire. L'enseignante explique quand et comment on fera de la grammaire dans son cours puis demande à l'ensemble du groupe « est-ce que c'est un problème la grammaire ». C'est Hamed qui répond et elle réitère donc sa question en précisant qu'elle s'adresse à tout le monde.

extrait 4 : (Viviane cours 1)

$198 \mathrm{P} \quad(\ldots)$ est-ce que c'est un problème la grammaire

199 Hamed bien sûr

$200 \mathrm{P} \quad$ pour tout le monde $\nearrow$ c'est vrai $\nearrow$ c'est un problème $\nearrow$

201 As <murmures>

202 Hamed il faut apprendre la grammaire chaque chaque chaque euh langue a grammaire $=$

$203 \mathrm{P}=\mathrm{ah} \mathrm{BON} \nearrow$

204 Hamed la grammaire française est difficile

$205 \mathrm{P} \quad<$ ironique $>$ c'est pas vrai <rire>

206 Hamed <en riant> je jure

207 P est-ce que vous avez des GROS problèmes en grammaire française + qu'est-ce qui vous cause des GROS problèmes +

$208 \mathrm{Na} \quad$ les prépositions

$209 \mathrm{P} \quad$ les prépositions \ $\mathrm{P}$ 'est un problème ça $\nearrow$ ouai $\nearrow$ pour tout le monde $\nearrow$

$210 \mathrm{Na} \quad$ oui et avec des verbes employés avec des prépositions

$211 \mathrm{P} \quad \mathrm{c}$ 'est à dire les verbes qui utilisent à de avec

$212 \mathrm{Na} \quad \underline{\mathrm{à} \mathrm{de}}$

$213 \mathrm{P} \quad$ on ne sait jamais c'est de est-ce que c'est à pouf ouai d'accord et puis ++ $+\left(9^{\prime \prime}\right)$

214 Hamed c'est fini〉

215 P ça suffit comme ça

216 Hamed c'est trop

217 P $\quad$ y a des gens que j'nai pas entendus / j'aimerais bien entendre tout le monde ++ mademoiselle votre prénom c'est quoi

C'est après l'intervention de Hamed en 202, intervention qui contrarie visiblement le scénario de circulation de la parole prévu par l'enseignante, que cette dernière glisse sur un plan ironique qui n'est pas perçu immédiatement par Hamed. En 205 elle exagère donc très fortement le ton de la surprise et accompagne son c'est pas vrai d'un petit rire. Ce faisant, elle crée elle aussi le 'décalage' que nous avons évoqué en 2.2 et dont nous avions avancé qu'il était susceptible d'ouvrir un nouvel espace relationnel en permettant aux locuteurs de ne plus intervenir du seul point de vue des rôles interactionnels définis par leurs statuts. Mais pour que cette mobilisation d'une autre facette de l'identité fonctionne pleinement, il faut qu'elle soit reconnue par l'interlocuteur. C'est ce que fait Hamed en 206 qui ne se contente pas de sourire de l'humour de l'enseignante mais entre dans le jeu et investit cet espace relationnel non didactique en intervenant lui-aussi de manière ironique. Hamed a certes pu exprimer en 
206 une autre facette de son identité que celle de son 'je' apprenant mais l'absence totale de feedback de l'enseignante en 207 qui s'adresse alors à l'ensemble du groupe pour reprendre le déroulement de son scénario initial de remue-méninge ramène chacun à son indentité statutaire d'enseignant ou d'étudiant. On peut certes considérer qu'en 206, toutes les conditions recensées par Moore et Simon (2002: 139) pour l'apparition d'une donnée saisissable semblaient réunies : "émergence chez l'apprenant d'un besoin d'apprendre et/ou de communiquer», «implication de l'apprenant dans l'interaction », «expression par l'apprenant de sa double identité. ». Mais l'enseignante, ramène chacun à ses rôles interactionnels statutaires et laisse le «je jure » de Hamed sans réponse. Elle ne lui offre donc ni l'étayage qui lui permettrait de confronter son énoncé au système de la langue cible et peutêtre de développer sa compétence linguistique, ni le feedback, ne serait-ce que par un sourire, qui lui permettrait de savoir si son intention de communiquer et sa tentative de poursuivre l'échange ironique initié par l'enseignante a atteint son but.

En 214, comme en 206, Hamed essaie encore de faire sortir une nouvelle fois l'échange de ses rails. Après un silence de 9 secondes, il propose en effet d'interpréter le silence comme un essoufflement des idées des étudiants : «(ça ?) suffit comme ça ». L'enseignante se contente de répéter ce qu'a dit Hamed avec une intonation de surprise et d'interrogation, et avec le ça initial, difficilement audible dans l'énoncé de Hamed. Plus qu'une véritable question, c'est un signal indiquant le caractère non recevable à ses yeux de l'intervention de Hamed que l'enseignante lance au groupe. Mais, poursuivant à la fois la logique de production (saisir toutes les occasions de parler) et la logique de négociation identitaire par l'humour et l'ironie dans laquelle il est engagé depuis le début du cours, Hamed surenchérit : «c'est trop », cette fois avec un sourire et une intensité plus faible qui indiquent probablement qu'il a conscience d'être en train d'atteindre les limites acceptables par son interlocutrice. L'enseignante, en 216, lui oppose une fin de non-recevoir en ne donnant aucun feedback verbal ou non-verbal à son intervention qu'elle ne peut pas ne pas avoir entendue. Le processus de déritualisation ayant atteint les limites de l'acceptable dans le contexte de ce cours, l'enseignante entreprend de 'reritualiser' les échanges. Probablement pour ne pas permettre une nouvelle initiative de Hamed et donner à l'ensemble du groupe la possibilité de « réintégrer » le cadre participatif, elle attribue explicitement la parole à une des apprenantes en justifiant ce changement du scénario didactique (abandon de la question à la cantonade) par une justification très professorale (j'aimerais bien entendre tout le monde). Les apprenants sont dès lors invités à se tenir à leur rôle (1). en répondant à l'injonction de l'enseignante «parlez » et (2). en " parlant pour parler » (Cicurel 1992), puisqu'elle les somme désormais tous successivement de prendre la parole, pas seulement pour qu'ils expriment leurs desideratas mais parce qu'elle veut les avoir tous entendus.

\section{Identité statutaire, identité complexe, la recherche d'un difficile équilibre}

Dans cette dernière partie, nous proposons d'étudier des offensives discursives de Hamed où celui-ci semble revendiquer son identité d'apprenant et même parfois se construire une image 'd'étudiant modèle' comme pour contre-balancer ses interventions «rebelles» qui font souvent dévier les scénarios prévus par les enseignantes. Mais nous verrons aussi que cette identité d'apprenant est elle-même rendue complexe par des conflits entre les comportements que Hamed pense être efficaces en termes d'apprentissage et ceux dont il pense qu'ils sont susceptibles de lui construire une image 'd'étudiant modèle' aux yeux des autres participants à l'échange.

\subsection{Apprenant expert / étudiant modèle}


L'exemple le plus caricatural de l'ambivalence de certains comportements de Hamed concerne l'interdiction d'employer les dictionnaires bilingues. Pour introduire l'énonciation de cette règle, Viviane prend Hamed à partie : « c'est quoi ça sur votre table $\nearrow$ (...) c'est un très beau dictionnaire / il est GROS il est BEAU il est magnifique » puis elle explique que l'emploi en sera interdit sauf dans quelques rares activité parce qu'il vaut mieux demander à l'enseignante le sens d'un mot que de « décrocher » du cours pour aller chercher un mot et se perdre tout seul dans son dictionnaire. Hamed lui coupe la parole (chevauchement long qui montre sa détermination) pour surenchérir et expliquer, non sans difficulté, qu'un autre désavantage de l'emploi du dictionnaire, est que pour un même mot, on trouve parfois plusieurs définitions. Cette intervention contribue à reconstruire l'image de bon étudiant mise à mal par le fait qu'il a servi de contre-exemple. En même temps, il nous semble important de souligner, pour bien montrer la complexité des identités d'apprenant, que deux fois dans ce cours là et deux fois dans le cours qui suit, Hamed utilisera son dictionnaire bilingue, suffisamment ostensiblement pour que cela donne lieu à des reproches de l'enseignante.

Cet emploi du dictionnaire s'inscrit dans une quête du sens qui semble très forte chez Hamed, et le conduit à interrompre régulièrement les activités pour demander des explications de vocabulaire qui sont parfois rallongées par le fait que Hamed ne supporte guère d'en rester à une compréhension approximative. Mais au delà de cette quête du sens des mots, il manifeste également une quête du sens des tâches, qui fait de lui un apprenant à la fois très impliqué et très critique. Il rejette ainsi une activité de groupe que l'enseignante justifie en disant que cela entraînera les apprenants à «échanger des idées ». Il argumente son rejet en précisant que la tâche n'est pas d'échanger des idées puisqu'il faut répondre à des questions de compréhension.

Dans l'extrait ci-dessous, on voit Hamed refuser de différentes manières la focalisation, initiée par l'enseignante, sur le sens des mots de la consigne pour opérer une focalisation sur le sens de la tâche proposée.

extrait 5 (Viviane cours 1)

\begin{tabular}{|c|c|c|}
\hline 306 & $\mathrm{P}$ & <elle écrit au tableau> qu'est-ce que c'est ça 〉 lexique personnel \\
\hline 307 & Hamed & lexique $>$ ça vient de Mexique peut-être \\
\hline 308 & $\mathrm{P}$ & $\begin{array}{l}\text { lexique ça vient de Mexique vous êtes d'accord } \nearrow \text { ça c'est cent pour cent } \\
\text { Hamed non } \searrow\end{array}$ \\
\hline 309 & As & $<$ rires $>$ \\
\hline 310 & $\mathrm{P}$ & désolée non〉 \\
\hline 311 & Hamed & $\mathrm{X}$ le vocabulaire personnal ça veut dire quoi $\nearrow$ \\
\hline 312 & $\mathrm{P}$ & pardon / le vocabulaire $\nearrow$ \\
\hline 313 & Hamed & ça veut dire quoi / le vocabulaire / personnal \\
\hline 314 & $\mathrm{P}$ & personNEL \\
\hline 315 & Hamed & personnel \\
\hline 316 & $\mathrm{P}$ & personNEL qu'est-ce que ça veut dire personnel` \\
\hline 317 & Hamed & c'est personnel / c'est à moi / le mienne \\
\hline 318 & $\mathrm{P}$ & exactement alors $=$ \\
\hline 319 & Hamed & $=$ ça veut dire quoi c'est \\
\hline
\end{tabular}

$320 \mathrm{P} \quad=c ̧ a$ veut dire QUOI \ j'vais vous poser ici <elle écrit au tableau les numéros $>$ six questions $\searrow$ où j'vais vous demander de choisir UN mot de la langue française $\rangle$ alors par exemple $\searrow$ la première $\boldsymbol{\lambda}\langle$ elle écrit $\rangle$ dans la 
langue française / quel est le mot que vous détestez \ chacun va donner sa réponse

321 Hamed un mot ou: le type le type de mot

$322 \mathrm{P} \quad$ vous comprenez ma question $\boldsymbol{\gamma}$ un mot $\searrow$ un exemple $\searrow$ quel est le mot que vous détestez

323 Hamed pourquoi mais de quelle façon $\nearrow$

$324 \mathrm{P} \quad$ d'accord un exemple $\nearrow$ un mot que je déteste moi en français c'est: la guerre

325 Hamed hm: d'accord

Dans cet extrait, Hamed apparaît comme un apprenant expert dans la mesure où il sollicite à 5 reprises $(311,313,319,321,323)$ de la part de l'enseignante les compléments d'information qui lui permettront de donner du sens à l'activité qu'elle propose.

Alors que l'enseignante, après avoir écrit le titre de l'activité Lexique personnel au tableau sollicite de la part des apprenants une explication littérale des mots écrits, Hamed donne une réponse fantaisiste, que l'enseignante bien entendu rejette. Peut-être lui fallait-il le temps des interventions $(308,309,310)$ pour retrouver le sens du mot lexique, toujours est-il que dès l'intervention 311, il renvoie à l'enseignante sa question. En remplaçant le mot lexique par vocabulaire, (en 311), il fait savoir indirectement à l'enseignante qu'il connait le sens du mot lexique et il tente de donner à la question qu'il pose une dimension non pas métalinguistique mais plutôt pédagogique. L'enseignante maintient la dimension métalinguistique de l'échange en proposant une correction de la prononciation de personnel (314) puis en demandant une définition de personnel (316). Hamed s'exécute (317) mais lorsque celle-ci produit le troisième temps de l'échange ternaire didactique en évaluant positivement sa réponse (318), il lui coupe la parole pour tenter de réitérer sa question sur le sens de l'activité qu'elle propose. Cette fois-ci, c'est elle qui l'interrompt pour donner un exemple de questions qu'elle va leur poser.

Mais cet exemple ne satisfait pas non plus Hamed car il pressent sans doute que plutôt qu'un mot de la langue française détesté, c'est un 'référent détesté' qu'elle leur demande de désigner par un mot français. L'exemple que donne l'enseignante en 324 vient d'ailleurs confirmer cette hypothèse puisque l'enseignante propose la guerre. L'emploi du déterminant la aussi bien que le choix du mot guerre oriente plutôt vers une interprétation de la consigne comme « dites-nous en français ce que vous détestez le plus ». Toutes les réponses que donneront les étudiants (souris, traître...) manifesteront d'ailleurs une telle interprétation de la question.

Dans sa quête du sens de l'activité proposée et dans son intuition de l'ambiguïté de la tâche demandée, Hamed nous apparaît comme un apprenant expert. Or paradoxalement, c'est cette expertise qui le conduit à avoir un comportement assez éloigné de celui d'un "étudiant modèle ». Il remplit à plusieurs reprises des rôles interactionnels qui ne sont pas ceux classiquement occupés par les étudiants dans l'interaction didactique. Il interrompt l'enseignante en 319. En 321, il se pose en expert en lui proposant une reformulation possible de ce qu'elle veut dire (un mot ou : le type le type de mot). Enfin, après l'exemple que donne l'enseignante en 324, la réponse de Hamed, produite sans aucune marque intonative d'ironie hm d'accord signifie aussi bien «j'ai compris » que «d'accord j'accepte de jouer le jeu ». Il informe ainsi indirectement que jusque-là, il n'était pas d'accord et il se pose en 'partenaire' de l'activité et non en simple exécutant.

\subsection{Locuteur expert / étudiant modèle}

On rencontre d'autres exemples de ce jeu de balancier comme dans cette séquence d'un cours de Dorie, qui interrompt, pendant 115 tours de parole, l'activité de compréhension orale d'un 
reportage télévisé sur les chiens et les chats. Ici c'est plutôt un conflit entre la volonté d'être reconnu à la fois comme «locuteur expert » et comme « "étudiant modèle » que l'on rencontre. L'enseignante y développe le thème des allergies introduit dans le document visionné en classe. Elle invite les apprenants à raconter leurs expériences d'allergies 159 : « est-ce que vous avez des allergies $\lambda$ les uns et les autres $\lambda$ ». Les apprenants se prêtent au jeu du partage d'expériences. Avant Hamed, Na parle des allergies à certains aliments, Senko explique qu'elle est allergique à la poudre des arbres mais pas aux chats, Yon Keï témoigne de son allergie à l'amande de noyau d'abricot dans les cosmétiques, Hamed explique quant à lui en 235 : «mon frère il est allergie au : de pêche » puis il précise en 251. «il touche / pas manger / il TOUche la pêche ».

Entre ces deux interventions répondant à la question initiale, on constate que tous les échanges sont consacrés à un travail métalinguistique, toujours à l'initiative de l'enseignant et dont aucune n'est motivée par un problème d'intercompréhension. Ces séquences parenthétiques métalinguistiques constituent donc un travail formel "en roue libre", dans la mesure où il ne contribue pas à la réalisation de la tâche en cours.

\section{Extrait 6 (Dorie cours 1)}

235. Hamed mon frère il est allergie au : de pêche

236. $\mathrm{P} \quad$ il est allergique à

237. Hamed X

238. $\mathrm{P} \quad$ redites / la phrase / votre frère oui

239. Hamed il est allergie

240. P il est allerGIQUE

241. Hamed aller allergique comme ça $\boldsymbol{\lambda}$

242. P oui

243. Hamed il A allergie

244. P alors il a UNE allergie

245. Hamed Ah

246. P à / la pêche

247. Hamed donc il a allergique

248. P il est allergique

249. Hamed au pêche

250. $\mathrm{P}$ à la pêche

251. Hamed à la pêche il touche / pas manger / il TOUche la pêche

La sollicitation initiale de Dorie, qui a demandé aux apprenants en 159 s'ils ont des allergies, semblait initier une séquence de type conversationnel. L'élément de réponse apporté par Hamed à cette sollicitation ne sera pris en compte comme constituant une avancée thématique qu'en 252. Entre les deux, l'avancée de l'échange conversationnel est comme suspendue et c'est à son rôle interactionnel d'apprenant que Dorie convoque Hamed dès l'intervention 236. Elle sollicite de lui des «quittances » de savoir (Py, 1990 : 87) par l'emploi d'un impératif, en 238 et par les accents didactiques des interventions 240 et 244 . Or Hamed ne se conforme pas sans réticence au rôle interactionnel qu'elle veut lui imposer. Il essaie de reprendre l'initiative de l'échange en 241, sans remettre en cause, à ce moment là, la dimension métalinguistique des échanges. L'emploi du donc en 247 (donc il a allergique) semble vouloir fonctionner comme une forme de bornage (Goffman dirait qu'il cherche à "fermer la parenthèse") de la séquence métalinguistique, pour replacer ses interventions sur un mode référentiel. En vain, car l'enseignante, en 248, poursuit le travail de correction formelle. Cette fois, Hamed ne donne pas de «quittance de savoir » et poursuit son avancée thématique pour finalement, en 251, parvenir à donner la suite de sa contribution à la discussion sur les allergies. En termes 
de territoire d'apprenant, il a donc choisi de privilégier le pôle tâche pour apporter lui-aussi une information 'sensationnelle' à la conversation et revendique ainsi d'être reconnu lui-aussi comme «locuteur-expert» de la conversation. Il se pose momentanément en tant que 'jepersonne' (cf. mon frère il) quitte à inhiber le 'je-étudiant' qui se devrait de se conformer au scénario interactionnel de l'enseignante visant à un travail métalinguistique.

Dans la séquence suivante, c'est Hamed au contraire qui prend l'initiative de l'ouverture d'une séquence métalinguistique, comme pour rehausser son image d'apprenant que la séquence précédente avait quelque peu mise à mal.

Extrait 7 (Dorie cours 1)

$252 \mathrm{P}$ ah oui oui c'est dangereux les allergies / on peut mourir d'une allergie / vous savez les les les guêpes / vous connaissez les guêpes $\boldsymbol{\nearrow}$ bzz bzz les petits insectes / qui piquent

253 Hamed ils s'appellent guêpe

$254 \mathrm{P} \quad$ une guêpe

255 Hamed comment s'appelle XX qui fait le miel c'est abeille 7

$256 \mathrm{P} \quad$ c'est l'abeille qui fait du miel

257 Hamed l'abeille

$258 \mathrm{P} \quad$ oui l'abeille qui fait du miel / pas la guêpe hein / une guêpe / c'est bon vous voyez là / une guêpe

259. Af $\mathrm{XX}$ abeille

260. $\mathrm{P} \quad$ oui un peu à part que le corps vous savez a des rayures noires et jaunes

261 Hamed mais l'abeille elle pique aussi

262. P l'abeille aussi pique / oui la guêpe / l'abeille / mais la guêpe est très dangereuse / on peut mourir avec une guêpe avec UNE piqûre de guêpe

263. Hamed comment s'appelle le plus grande / la guêpe plus grande

264. $\mathrm{P} \quad$ la reine $\boldsymbol{\lambda}++$ le bourdon ou un bourdon $\nearrow$

265. Hamed oui oui comme ça X

266. P le bourdon $\nearrow$ oui une grosse guêpe oui

267. Hamed XXX (le bourdon?)

Hamed développe ainsi des activités d'apprentissage variées, en gardant toujours l'initiative. En 253, il reprend en mention le mot guêpe que Dorie veut leur enseigner. En décontextualisant le mot, il offre une forme de collaboration à l'activité d'enseignement que développe $\mathrm{P}$ et il développe explicitement l'activité d'apprentissage que sollicite P. Il en fait de même en 259 avec abeille. Il vérifie (ou il exhibe ?) sa compréhension du terme abeille en 255 et 261 et enfin, il sollicite un mot nouveau supplémentaire en 263. On peut se demander si cette focalisation sur le pôle norme n'a pas avant tout une fonction figurative. En se conformant à ce qu'il pense être le comportement d'un étudiant modèle (montrer ce que l'on sait, montrer sa motivation à apprendre et son intérêt pour les informations données par l'enseignant), Hamed chercherait ainsi à réparer le « coup de force » de l'extrait précédent.

\section{Conclusion}

Les comportements « remarquables » d'Hamed se regroupent donc autour de trois axes : -renégocier la relation pour préserver un espace relationnel 'ouvert' au delà des limites marquées par les rôles interactionnels statutaires des uns et des autres. Et de ce point de vue, on peut remarquer que ces comportements sont plus fréquents et plus marqués avec l'enseignante qui définit le rapport de place le plus étroitement didactique, à savoir Viviane. 
-se comporter conformément à ce qu'il pense bon pour son apprentissage. Il s'agit d'une part de saisir toutes les occasions de prendre la parole pour solliciter plus ou moins directement des données saisissables et se donner des occasions d'utiliser ses compétences langagières. Hamed s'inscrit alors sans le savoir, dans la logique de ce que Swain appelle l'hypothèse de la sortie (output hypothesis). Celle-ci considère notamment que c'est lorsqu'ils produisent que les apprenants ont l'occasion "d' 'étirer' leur interlangue de manière à atteindre des buts communicatifs »(Swain, 2000: 99). Il s'agit également d'autres types de comportements comme la quête du sens des activités didactiques proposées, ou l'utilisation d'outils interdits dans la classe (le dictionnaire).

-construire malgré tout une image d'étudiant modèle.

$\mathrm{Au}$ cœur de ces comportements remarquables se situe donc bien un travail d'appropriation langagière. Les comportements du premier axe apparaissent comme une condition pour qu'un travail d'appropriation puisse se développer. En effet, surtout dans le cas où les interactions de la classe sont très dirigées et rythmées par des questions didactiques, l'ouverture d'un espace relationnel extra-didactique apparaît comme une condition nécessaire pour que d'autres échanges se développent. Les comportements du troisième axe ont quasiment une valeur compensatoire par rapport à ceux des deux premiers. Cette volonté, manifestée sporadiquement mais régulièrement d'être reconnu comme un 'étudiant modèle' révèle que la déritualisation des échanges, même motivée par un projet d'appropriation langagière, peut se révéler coûteuse pour l'apprenant en termes d'image et qu'en classe, il peut être plus « confortable » de se comporter comme un étudiant modèle que comme un apprenant expert.

La question des images d'apprenant et d'étudiant que Hamed construit dans l'interaction n'a été abordée que succinctement et mériterait d'être approfondie. Il se pose en expert pragmatique. En effet, il se montre capable de monopoliser l'attention de l'enseignante en devenant son interlocuteur privilégié pendant de longues séquences. Il est également capable de faire sortir, avec plus ou moins de bonheur, l'échange didactique de son lit, notamment en faisant rire la classe. Il est capable enfin de jouer en français avec les mots et les noms propres (les exemples sont nombreux). A côté de cette expertise affirmée, il se comporte comme un apprenant conscient de sa 'marge de progression' du point de vue du vocabulaire et de la phonétique. Il sollicite des données saisissables et effectue des prises multiples dans ces deux domaines. Son attitude vis à vis de la syntaxe semble plus ambiguë. Il reconnaît explicitement que la grammaire constitue une difficulté et son problème avec l'écrit qu'il explicite également n'est peut-être qu'une autre facette de ses difficultés grammaticales. Mais dans les échanges, il marque peu d'hésitation sur des questions de morpho-syntaxe, il ne sollicite guère d'aide sur ce plan et n'opère que peu de prises dans les données, parfois explicitement correctives, fournies par les enseignantes. Il y a, dans ce portrait schématique du profil d'interactant-apprenant de Hamed, des pistes à explorer pour aborder d'une autre manière les articulations possibles entre construction d'images et travail d'appropriation.

Arditty, J. \& M.-T. Vasseur 1999. Interaction et langue étrangère, présentation, Langages $\mathrm{n}^{\circ} 134,3-19$.

Bange, P. 1992. A propos de la communication et de l'apprentissage de L.2, AILE $\mathrm{n}^{\circ} 1,53-85$

Bigot, V. 2002a. Les comportements langagiers tutélaires des enseignants, réflexion sur la mise en discours des activités cognitives des apprenants, In Cicurel F \& D. Véronique (eds) Discours et appropriation des langues, Presses de la Sorbonne nouvelle, 67-86. 
BIGOT, V. 2002b. Lieux et modes de construction de la relation interpersonnelle en contexte didactique : analyses d'interactions verbales en cours de français langue étrangère. Thèse de Doctorat sous la direction de Francine Cicurel, Université Paris 3.

Krafft, U. \& U. Dausendschön-Gay 1994 «Analyse conversationnelle et recherche sur l'acquisition » Bulletin VALS/ASLA 59, 127-158.

Cicurel, F. 1992. Le canevas didactique de production discursive, Intercompreensão, Revista de didáctica das Línguas 2, Santarem, Portugal, 9-21

Fillietaz, L. 2005. Mise en discours de l'agir et formation des enseignants. Quelques réflexions issues des théories de l'action. Le Français dans le monde, Recherches et applications, juin 2005.

Grandcolas, B. 1980. La communication dans la classe de langue. FDM 153, 53-57.

Kerbrat-Orecchioni, C. 1990, 1992. Les interactions verbales I et II, Didier.

Mondada, L \& S. Pekarek 2001. Interactions acquisitionnelles en contexte, FDM Recherches et Application, 107-137.

Mondada, L \& L. Gajo 2000. Interactions et acquisition en contextes, Fribourg, Editions universitaires de Fribourg.

Moore, D. \& D.L. Simon 2002. Déritualisation et identité d'apprenants. AILE n 16, 121-144.

Py, B. 1990. Les stratégies d'acquisition en situation d'interaction. Le Français dans le Monde, Recherche et Applications, fév-mars 1990.

Py, B. 1993. L’apprenant et son territoire : système norme et tâche, $A I L E \mathrm{n}^{\circ}$ 2, 9-24.

Swain, M. 2000. The output hypothesis and beyond: Mediating acquisition through collaborative dialogue. In J.P. Lantolf (Dir), Sociocultural Theory and Second Language Learning, Oxford University Press.

We examine here the manner in which an adult student, whose language classrooms lessons where video-taped, take interactional initiatives that change the course of the verbal exchanges and the teacher's scenario of the lesson. The examples analysed show how the student, through the student's discursive offensive, renegociate the interpersonal teacherstudent relationship and redefine his «learning territory » so that his language producing opportunities are increased in a collaborative context where the teacher's scaffolding helps his communicative abilities to develop.

Key words : classroom discourse ; interpersonal relationship ; L2 learning.

\section{Pour citer cet article :}

Violaine Bigot, «Négociation de la relation et processus d'appropriation en classe de langue », Acquisition et interaction en langue étrangère $\mathrm{n}^{\circ} 22$, p.17-42.

[En ligne], 22 |2005. URL : http://journals.openedition.org.ezproxy.univ-paris3.fr/aile/1716 